\title{
Cognitive Impairment Secondary to Treatment of Cured Brain Metastases of Triple-Negative Breast Carcinoma
}

\author{
Nassif E*, Arsene-Henry A, Kirova YM \\ Department of Radiotherapy, Institut Curie, 26 rue d'UIm, 75005 Paris, France
}

\section{*Corresponding author:}

\section{Elise Nassif}

Department of Radiotherapy,

Institut Curie, 26 rue d'Ulm, 75005 Paris, France

Received : April 12, 2019

Published: May 2, 2019

\section{ABSTRACT}

Context: Brain metastases from triple-negative breast cancer (TNBC) are not curable and can be invalidating, rapidly progressive. This justifies aggressive treatments.

\begin{abstract}
Materials and methods: We report the case of a 58 years old women cured of brain metastases from TNBC. Initial diagnosis was of locally advanced inflammatory TNBC for which the patient underwent high-dose neo-adjuvant chemotherapy followed by surgery, radiotherapy and adjuvant chemotherapy. Symptomatic brain metastases were diagnosed 10 months after the end of adjuvant chemotherapy. Whole-brain radiotherapy and stereotactic radiosurgery were rapidly delivered followed by twelve months of chemotherapy.

Results: The patient is still alive and disease-free twelve years after these aggressive treatment protocols. However, she is disabled from neurologic and cognitive toxicities from treatments. There is currently no available treatment of this cognitive deterioration. Preventive measures are scarce and some are under study with promising results. The multiplicity of neurotoxic treatments for patients with brain metastases should be avoided when possible. The benefit-risk balance should be closely evaluated.
\end{abstract}

\section{CLINICAL CASE}

We report the case of a 58 years-old woman with diagnosis of localized inflammatory left triple negative breast cancer (TNBC) in September 2003. Initial treatment consisted of neo-adjuvant high-dose chemotherapy with four cycles of epirubicin and cyclophosphamide. On December 2003, total mastectomy with axillary lymph node dissection was performed. Pathological evaluation found a $20 \mathrm{~mm}$ residual tumor with one metastatic axillary lymph node. Adjuvant radiotherapy of the left thoracic wall and all lymph node areas was performed. Four cycles of adjuvant chemotherapy with docetaxel and 5-Fluorouracil were administered. This treatment protocol ended in June 2004.
In April 2005, the patient reported headaches, vertigo, left arm tremors and mnesic dysfunction. Two temporal masses were found, measuring $36 \mathrm{~mm}$ and $20 \mathrm{~mm}$. High dose intravenous steroids were started, with anti-epileptic medication valproate. Whole brain radiotherapy (WBRT) was performed ending in May 2005. Rapid stereotactic radiosurgery (SRS) was performed in June 2005. Adjuvant treatment consisted of twelve cycles of oral capecitabine.

Neurologic symptoms started to exacerbate in September 2005 , during the adjuvant course of treatment. Initial reports of neurologic deterioration consisted of imbalance problems and extra-pyramidal syndrome. The latter symptom was linked to the valproate treatment by anti-dopaminergic toxicity and successfully reversed when valproate treatment was switched 
for carbamazepine. In September 2005, an epileptic seizure revealed a right thalamic hematoma secondary to a posttherapeutic cavernoma. Bilateral tinnitus and unexplained urinary incontinence are now constant. Increasing imbalance disorders have impacted the patient's quality of life and a wheelchair has been necessary since 2013.

Cognitive impairment is the most disabling handicap reported by the patient, consisting of anterograde amnesia, frontal disinhibition, attention deficit and multiple dyspraxia. After extensive clinical and radiological evaluation, symptoms have been linked to post-therapeutic leukoencephalopathy (Figure 1). Treatment is rehabilitation and alleviation of psychiatric medication, as the patient has been under various antidepressants and benzodiazepine medication for decades. A small benefit has been noted, albeit modest.

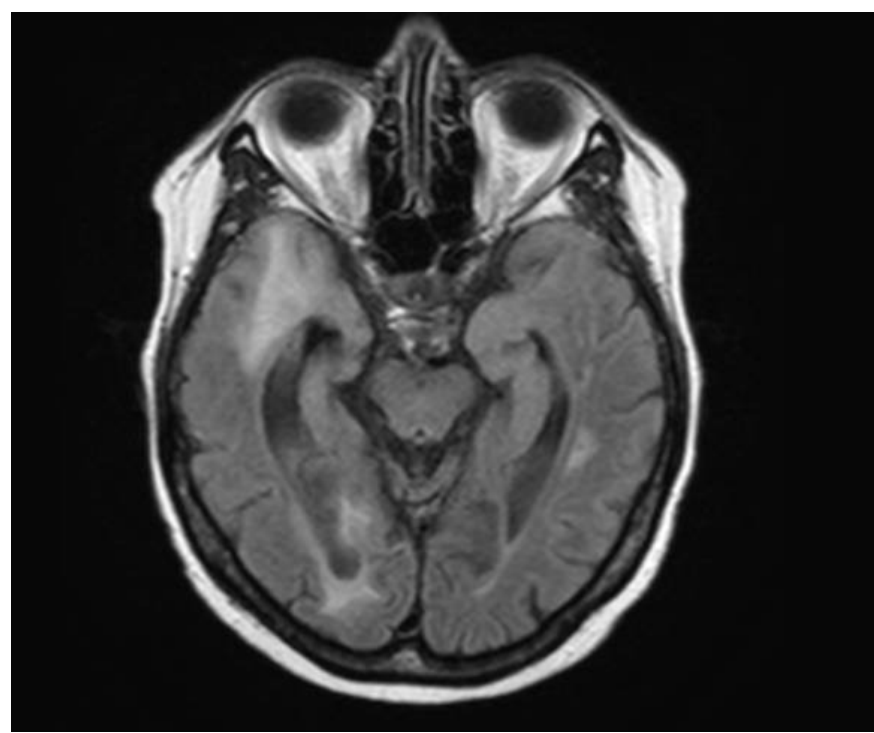

Figure 1: $2016 \mathrm{MRI}$, leukoencephalopathy

\section{DISCUSSION}

We report the case of a cured patient twelve years after the end of all oncologic treatments for brain metastases (BM) of TNBC. The survival benefit is not debatable, since this patient presented with advanced stage highly aggressive disease. However, at 72 years-old, she is now disabled secondary to cognitive toxicity. The benefit-risk balance of treatments should be questioned.

Several risk factors for cognitive impairment can be found and their multiplicity probably acts in a synergistic manner with oncological treatments. Steroids act on neurogenesis and neural plasticity. A role of anaesthetic drugs in cognitive deterioration has been suggested. Epileptic seizures are responsible for neuronal changes, and epileptic medication reduces neurological processing speed. Age and comorbidities are common risk factors of cancer and cognitive decline.

WBRT is one of the major causes of cognitive toxicities reported after BM treatment[1]. Special attention has been paid towards the mechanisms underlying this toxicity[2]. Rapid SRS after WBRT should be avoided, as the risk of radionecrosis is increased. Chemotherapy can cause cognitive toxicities[3], though not all protocols are equal. High dose chemotherapy is more toxic than standard chemotherapy[4]. Likewise, anthracycline-based regimens cause more cognitive deterioration[5]. Other oncological treatments, not present here, such as hormone therapy or certain targeted therapies impair cognition but have been poorly studied.

Thirty per cent of patients treated for cancer will eventually develop BM. Up to $50 \%$ of these patients will live up to encounter cognitive toxicities. Attention should be drawn towards cognitive impairment following treatment of BM[6]. Every medical treatment decision should be tailored to the patient's own cognitive risk. Furthermore, trials are needed to properly assess cognitive toxicity of specific oncological treatments and curative as well as preventive measures.

\section{REFERENCES}

1. Welzel G, Fleckenstein K, Schaefer J, Hermann B, KrausTiefenbacher U, Mai SK, et al. (2008) Memory function before and after whole brain radiotherapy in patients with and without brain metastases. Int J Radiat Oncol Biol Phys 72(5): 1311-8.

2. Makale MT, McDonald CR, Hattangadi-Gluth JA, Kesari S (2017) Mechanisms of radiotherapy-associated cognitive disability in patients with brain tumours. Nat Rev Neurol 13(1): 52-64.

3. Ahles TA, Saykin AJ (2007) Candidate mechanisms for chemotherapy-induced cognitive changes. Nat Rev Cancer 7(3): 192-201.

4. Schagen SB, Muller MJ, Boogerd W, Rosenbrand RM, van Rhijn D, Rodenhuis S, et al. (2002) Late effects of adjuvant chemotherapy on cognitive function: a follow-up study in breast cancer patients. Ann Oncol 13(9): 1387-97.

5. Kesler SR, Blayney DW (2016) Neurotoxic Effects of Anthracycline-vs Nonanthracycline-Based Chemotherapy on Cognition in Breast Cancer Survivors. JAMA Oncol 2(2): 185-92.

6. Franchino F, Ruda R, Soffietti R (2018) Mechanisms and Therapy for Cancer Metastasis to the Brain. Front Oncol 8: 161.

Copyright: Nassif E, et al. @2019. This is an open-access article distributed under the terms of the Creative Commons Attribution License, which permits unrestricted use, distribution, and reproduction in any medium, provided the original author and source are credited.

Citation: Nassif E (2019). Cognitive Impairment Secondary to Treatment of Cured Brain Metastases of Triple-Negative Breast 